$87.5 \%$ of the patients completing the study had repeat MRI scans. Of those, $85.7 \%$ were found to have no new or gadoliniumenhancing lesions.

JC Virus antibody testing was performed after two years of NAT. Of the thirteen samples, eight $(61.5 \%)$ tested positive. Two of those (25\%) discontinued NAT due to previous IV mitoxantrone treatment. The remaining patients continued treatment under close supervision by the attending neurologist.

No cases of Progressive Multifocal Leukoencephalopathy were reported.

Conclusions Long-term therapy with natalizumab proved to be safe and effective in our population. Strict follow-up criteria were implemented for JCV antibody-positive patients remaining on treatment with natalizumab for more than two years.

No conflict of interest.

\section{CPC-092 NEUROPSYCHOLOGY OF SAUDI COLON CANCER PATIENTS}

doi:10.1136/ejhpharm-2013-000276.549

'N Abanmy, ${ }^{2 P}$ Gard, ${ }^{2}$ A Macadam, '0 Alomran. 'King Saud University, Clinical Pharmacy, Riyadh, Saudi Arabia; 'University of Brighton, Pharmacology and Therapeutics, Brighton, UK

Background Colorectal cancer is a common disease and its prevalence is second to that of breast cancer worldwide. In Saudi Arabia the disease is ranked second after breast cancer and accounts for $8.5 \%$ of all tumours. Evaluation of Quality of life (QOL), anxiety and depression of such patients, as well as neurocognitive properties, is important to assess the impact of both malignancy and/or exposure to treatments including chemotherapy and surgery.

Purpose To assess the neuropsychology of a group of Saudi colon cancer patients 6 months after treatment was completed.

Materials and Methods Patients (18- 60 years) were recruited from the oncology clinic at King Khaled University Hospital (KKUH) at Riyadh, the capital city of Saudi Arabia. Exclusion criteria included smoking, psychiatric or cerebrovascular disease, sensory impairment, abnormal electrolytes, anaemia or uncontrolled blood pressure. Healthy volunteers were randomly recruited from the same hospital, however the availability of matched age controls was difficult. Cognition was assessed using the Rey Auditory-Verbal Test RAVLT (learning \& memory); the Rey-Osterrieth complex figure (RCF, visuo-spatial organisation and visuo-spatial memory); semantic verbal fluency (executive function); letter cancellation (attention); digit-symbol (sustained attention, visual searching, visual sequencing). The Arabic version of 36-item Short-Form Health Survey SF-36 and the Hospital Anxiety and Depression Scale (HADS) were also used to assess QOL, anxiety, and depression, respectively.

Results A total of 32 colon cancer patients in remission were recruited, their mean age was 44.8 years. 23 of them were males $(71.9 \%)$, while their mean years of education was $13.1 \pm 4.06$ years. Healthy controls $(n=36)$, were significantly younger than the patients (34 Years) $(\mathrm{t}(66)=-4.2, \mathrm{P}<0.05)$. There were no differences between the groups in terms of QOL, anxiety, depression, attention, executive function, oral and visuospatial memory. Healthy controls had significantly better RCF recall task $(\mathrm{t}(67)=2.61, \mathrm{p}<0.01)$ and delayed recall task $(\mathrm{t}(67)=3.16$, $\mathrm{p}=0.002)$ than colon cancer patients.

Conclusions This study indicates that neither colon cancer, nor its treatment, has any significant impact on the psychological wellbeing of the patients in comparison to healthy controls. The significant differences in recall may reflect the differences in age between the groups.

No conflict of interest.

\section{CPC-093 NOSOCOMIAL INFECTIONS IN A COHORT OF EXTRA-CORPOREAL LIFE SUPPORT PATIENTS}

doi:10.1136/ejhpharm-2013-000276.550

'D Lenehan, 'N Scanlon, ${ }^{2}$ Conrick-Martin, ${ }^{3} \mathrm{~J}$ O'Gorman, ${ }^{2} \mathrm{D}$ Oshodi, ${ }^{2} \mathrm{~S}$ O'Brien, ${ }^{3} \mathrm{M}$ Hannan, ${ }^{3} \mathrm{M}$ Lynch, ${ }^{2} \mathrm{E}$ Carton, ${ }^{1} \mathrm{C}$ Meegan. ${ }^{\prime} \mathrm{MMUH}$, Pharmacy, Dublin, Ireland (Rep.); ' ${ }^{2} \mathrm{MMUH}$, Intensive Care Unit, Dublin, Ireland (Rep.); ${ }^{3} \mathrm{MMUH}$, Microbiology, Dublin, Ireland (Rep.)

Background Extra-Corporeal Life Support (ECLS) is a method of life support used to treat patients with severe respiratory and/or cardiac failure refractory to conventional modes of treatment. Nosocomial infections in these patients are associated with increased morbidity and mortality along with increased lengths of intensive care unit (ICU) and hospital stay. No international best-practise consensus guidelines exist for treatment and/or prophylaxis of infections in this patient group.

Purpose To examine the rate of nosocomial infection in $\mathrm{MMUH}$ ECLS patients as well as the consumption of antimicrobials in the treatment and prevention of these infections.

Materials and Methods In a retrospective cohort study, the pharmacy records from a daily multi-disciplinary microbiology round reviewed all patients who are on ECLS. The use of prophylactic and therapeutic antimicrobials in these patients was assessed as well as the background ICU bloodstream infection rate.

Results Data analysis yielded a total of 17 patients over a two-year period, with a total of 444 ECLS days. In total, there were 17 infections in this cohort including $4(24 \%)$ blood-stream infections (yielding a rate of 9.0 per 1000 ECLS days). The first four ECLS patients received antibacterial (vancomycin) and antifungal (caspofungin) prophylaxis for the duration of ECLS, whereas the later cohort of 13 did not. In the cohort of patients who received prophylactic antimicrobials, defined daily doses (DDDs) per 100 ECLS days for vancomycin and meropenem were 49.54 and 49.63 respectively. For the non-prophylaxis cohort this was 25.31 and 37.73 respectively.

Conclusions The infection rate in this cohort was low. In particular, the bloodstream infection rate compared favourably with previously published rates, and was comparable with the 'background' bloodstream infection rate of the ICU population as a whole. Antimicrobial use in ECLS patients was high relative to overall ICU antimicrobial use.

No conflict of interest.

\section{CPC-094 NUTRITIONAL STATUS OF HOSPITALISED PATIENTS WITH HEAD AND NECK CANCER}

doi:10.1136/ejhpharm-2013-000276.551

C Vázquez López, M Suárez Santamaría, M Alvarez Payero, D Pérez Parente, MS Ucha Samartín, G Piñeiro Corrales. Meixoeiro Hospital (CHUVI), Pharmacy, Vigo, Spain

Background Malnutrition is common in hospitalised patients with head and neck carcinoma.

Purpose The aim of this study was to analyse the causes of hospitalisation of patients with head and neck cancer and to evaluate the nutritional status; type and route of nutrition therapy during hospitalisation and at discharge.

Materials and Methods Retrospective study of patients with head and neck cancer, between October 2011 and March 2012 at 420-beds hospital.

We examined demographic data, cause of admission to hospital, type and location of tumour, nutritional status before admission and at discharge by CONUT $^{\circledR}$ (system for early detection and monitoring of clinical undernutrition based on biochemical parametres and immune indicator) and type of nutritional therapy. We used the data source as medical record (IANUS ${ }^{\circledR}$ ). 
Results Were included 13 patients, with a mean age of $67 \pm 13.5$ (39-86). The location of tumour was 6 in oral cavity and 7 in oropharynx. The causes of admission was surgery (69.3\%) and complications of neoplastic pathology base (39.7\%); bleeding: $40 \%$, dysphagia: $20 \%$ bronchoaspiration: $20 \%$, oral mycosis: $20 \%$.

According to the nutritional status before admission, were found 4 patients $(30.7 \%)$ with mild malnutrition, $2(15.3 \%)$ with moderately malnutrition, $1(7.7 \%)$ with severe malnutrition and 6 patients with not available data. At discharge: 5 patients (38.5\%) with mild malnutrition, 7 patients with not available data and one patient died during the period.

During the admission period, all the patients received oral feeding, 6 patients received enteral nutrition (EN) by gastrostomy tube. At discharge, $61.5 \%$ of patients received oral diet and the $7.7 \%$ of them needed energy supplementation. The remaining 30.8\% needed to continue with EN.

Conclusions The risk of malnutrition in patients with head and neck cancer is high.

Individualized nutritional support in these patients is necessary to prevent weight loss.

In the absence of parameters to perform an adequate nutritional assessment, we need greater involvement by hospital physicians with the clinical nutrition unit.

No conflict of interest.

\section{CPC-095 OFF-LABEL USE OF ANAKINRA IN A PATIENT WITH FAMILIAL MEDITERRANEAN FEVER: A CASE REPORT}

doi:10.1136/ejhpharm-2013-000276.552

'F Valiente, 'I Sánchez, ${ }^{2}$ A Martínez-Blázquez, 'MA Rodríguez, '1 Ramos. 'Hospital de la Vega Lorenzo Guirao, Pharmacy, Murcia, Spain; ${ }^{2 H o s p i t a l ~ d e ~ l a ~ V e g a ~ L o r e n z o ~ G u i r a o, ~}$ Internal Medicine, Murcia, Spain

Background Familial Mediterranean Fever (FMF) is an autosomal recessive autoinflammatory disease characterised by periodic episodes of fever, peritonitis, arthritis and may be complicated by secondary amyloidosis. FMF affects groups of people from around the Mediterranean Sea. Colchicine is the standard treatment in the prevention of both acute attacks and secondary amyloidosis but there are some resistant patients. Anakinra, an interleukin-1 (IL-1) receptor antagonist indicated for the treatment of the signs and symptoms of rheumatoid arthritis in combination with methotrexate, is also known to affect the severity and the frequency of FMF attacks. Purpose To describe the progress of a patient with FMF treated with anakinra as IL-1 blocker, and evaluate the efficacy and safety of this treatment

Materials and Methods We describe the case of a 53-year-old colchicine-resistant woman suffering from FMF, who was treated with anakinra between April and September 2012 as second-line treatment, after several episodes of recurrent fever and abdominal pain. In order to evaluate the treatment the patient's clinical history and analytical data (C-reactive protein) were reviewed.

Results Anakinra was started with a daily subcutaneous dose (100 mg) associated with oral corticosteroids (methylprednisolone $8 \mathrm{mg}$ ). After the first cycle of treatment, the patient was fine, with no recurrent episodes of fever or abdominal pain. C-reactive protein (CRP) fell from 0.8 to $<0.1 \mathrm{mg} / \mathrm{dl}$. There were no injection site reactions. The only noteworthy adverse effect was neutropenia $(1.4 \times 109 / \mathrm{L})$.Corticosteroids and anakinra doses were reduced to zero and $100 \mathrm{mg}$ every other day respectively.

Conclusions In this case of FMF, anakinra successfully suppressed the number of attacks and the symptoms, without significant adverse reactions and with improvement in quality of life. Controlled trials are necessary to confirm the safety and efficacy of interleukin-1 antagonists in FMF patients.

No conflict of interest.

\section{CPC-096 OPTIMIZATION OF THE TREATMENT OF RHEUMATOID ARTHRITIS WITH BIOLOGICAL TREATMENT}

doi:10.1136/ejhpharm-2013-000276.553

D Ferràndez Martí, A Camacho Calvente, C Campabadal Prat, RM Parés Marimon, R Sala Robinat, J Serrais Benavente, A Perelló Juncà. Hospital Igualada, Pharmacy, Barcelona, Spain

Background The introduction of biological treatment (BT) in the treatment of rheumatoid arthritis (RA) has led to better control of this disease, but on the other hand to a great increase in pharmacy costs.

Purpose To review BT regimens in patients with RA in order to optimise treatment; to try to increase the dosing interval in patients who are responding well and evaluate the savings made.

Materials and Methods Interventional prospective study aiming at optimising the treatment of RA with BT by expanding interval in patients with a good response: adalimumab $40 \mathrm{mg} /$ every 14-21 days and etanercept $50 \mathrm{mg} /$ every 10-14 days. The review of treatments was made jointly between the pharmacy and rheumatology, adjusting the dose and calculating the cost avoided.

Results Patients chosen to extend the dosing interval had a mean DAS28 value of 2.183 (DAS28 $<2.4$ is considered to mean disease remission). By extending the dosing interval $€ 108,049.47$ was saved in a year.

Conclusions The review and optimization of BT dosage regimens in RA patients in remission allowed us to control the disease and save money.

\section{Abstract CPC-096 Table 1}

Rheumatoid arthritis

\begin{tabular}{ll}
\hline Patients with BT & Extended interval \\
\hline Adalimumab: 62 patients & 10 patients $(16.1 \%)$ \\
Etanercept: 53 patients & 5 patients $(9.5 \%)$ \\
\hline
\end{tabular}

Etanercept (cost per unit: 236,805€)

\begin{tabular}{|c|c|c|c|c|}
\hline Posology & $\begin{array}{l}\text { Cost/patient/ } \\
\text { year }\end{array}$ & $\begin{array}{l}\text { Incremental } \\
\text { cost }\end{array}$ & $\begin{array}{l}\text { Number of } \\
\text { patients with } \\
\text { extended interval }\end{array}$ & $\begin{array}{l}\text { Annual } \\
\text { savings }\end{array}$ \\
\hline $\begin{array}{l}\text { Etanercept } 50 \mathrm{mg} / 7 \text { days } \\
\text { (standard) }\end{array}$ & $€ 12,313.86$ & & & \\
\hline Etanercept $50 \mathrm{mg} / 10$ days & $€ 8,524.98$ & $-€ 3,788.88$ & 2 & $€ 7,577.76$ \\
\hline Etanercept $50 \mathrm{mg} / 14$ days & $€ 6,156.93$ & $-€ 6,140.35$ & 3 & $€ 18,470.79$ \\
\hline \multicolumn{5}{|c|}{ Adalimumab (cost per unit: $514,145 €$ ) } \\
\hline Posology & $\begin{array}{l}\text { Cost/patient/ } \\
\text { year }\end{array}$ & $\begin{array}{l}\text { Incremental } \\
\text { cost }\end{array}$ & $\begin{array}{l}\text { Number of } \\
\text { patients with } \\
\text { extended interval }\end{array}$ & $\begin{array}{l}\text { Annual } \\
\text { savings }\end{array}$ \\
\hline $\begin{array}{l}\text { Adalimumab } 40 \text { mg/14 } \\
\text { days (standard) }\end{array}$ & $€ 13,567.77$ & & & \\
\hline $\begin{array}{l}\text { Adalimumab } 40 \text { mg/21 } \\
\text { days }\end{array}$ & $€ 8,946.13$ & $-€ 4,621.64$ & 6 & $€ 27,729.84$ \\
\hline $\begin{array}{l}\text { STOP upon prolonged } \\
\text { remission of the disease }\end{array}$ & $€ 13,567.77$ & $-€ 13,567.77$ & 4 & $€ 54,271.08$ \\
\hline
\end{tabular}

No conflict of interest.

\section{CPC-097 OPTIMIZING CLINICAL PHARMACY: DETERMINING CRITERIA TO TARGET "HIGH RISK" PRESCRIPTIONS}

doi:10.1136/ejhpharm-2013-000276.554

M Philippe, B Leroy, S Coursier, M Bourdelin, H Bontemps. L'hôpital Nord Ouest, Villefranche sur Saône, France

Background Because pharmacists do not yet systematically analyse prescriptions closely due to lack of time and resources, tools to optimise pharmaceutical validation must be developed. 